\title{
Inteligência e Contágio Emocional: um estudo com trabalhadores brasileiros e angolanos
}

\author{
Sônia Maria Guedes Gondim* \\ Universidade Federal da Bahia, Salvador, Brasil \\ Valdiney Veloso Gouveia \\ Universidade Federal da Paraíba, João Pessoa, Brasil \\ Gisele Debiasi Alberton \\ Ana Célia A. Simões \\ Franciane Andrade de Morais \\ Universidade Federal da Bahia, Salvador, Brasil \\ María Isabel Hernández Díaz \\ Instituto Politécnico de Tecnologias e Ciências (ISPTEC), Luanda, Angola
}

\begin{abstract}
RESUMO
O estudo comparou trabalhadores brasileiros e angolanos em relação à inteligência emocional e ao contágio de emoções, considerando função gerencial, sexo e nível educacional. Participaram 431 trabalhadores, sendo 300 do Brasil e 131 de Angola, 71,5\% com ensino superior, 37,8\% exercendo função gerencial e idade média de 30 anos. Utilizou-se um survey eletrônico. Os resultados indicaram diferenças entre os países quanto à inteligência, mas não ao contágio. No Brasil, mulheres e gestores obtiveram médias mais elevadas de inteligência emocional, e em Angola, mulheres com ensino superior apresentaram maiores médias de inteligência emocional. Em relação ao contágio de emoções positivas, mulheres gestoras com ensino superior se contagiam menos que homens na mesma posição. Encontraram-se correlações positivas entre inteligência emocional e contágio.

Palavras-chave: inteligência emocional; contágio emocional; sexo; gestão.
\end{abstract}

\section{ABSTRACT \\ Emotional Intelligence and Contagion: A study with Brazilian and Angolan workers}

This study compared Brazilian and Angolan workers in emotional intelligence and contagion, considering their managerial position, sex, and educational level. Participants were 431 workers, 300 from Brazil and 131 from Angola, 71.5\% with a higher education, 37.8\% in a management position, and a mean age of 30 years. They answered an electronic questionnaire. Results indicate there are differences in emotional intelligence, but no differences were found for contagion. In Brazil women and managers had higher average of emotional intelligence mean while in Angola women with a higher education had higher average of emotional intelligence. For the contagion of positive emotions, women managers with a higher education were less contagioned than men managers. The results showed positive correlations between emotional intelligence and contagion.

Keywords: emotional intelligence; emotional contagion; sex; management.

A regulação emocional constitui um dos temas de crescente importância no estudo das emoções (Barbalet, 2011; Campos, Walle, Dahl, \& Main, 2011;
Gross \& Barret, 2011; Tamir, 2011), inclusive no ambiente de trabalho (Hochschild, 1983; Nelis, Quoidbach, Hansenne, \& Mikolajczak, 2011). A regu-

* Endereço para correspondência: Sônia Maria Guedes Gondim - sggondim@gmail.com 
lação, de si e do outro (Niven, Totterdell, Stride, \& Holman, 2011), é uma das dimensões da inteligência emocional (IE) (Mayer, Caruso, \& Salovey, 1999) e da competência emocional (Boyatzis, Goleman, \& Rhee, 1999), sendo bastante valorada no ambiente laboral, especialmente por seus impactos nas interações sociais e no bem-estar das pessoas envolvidas.

O contágio emocional (CE) é outro construto que se encontra relacionado com a inteligência, a regulação e as competências emocionais. Evidências empíricas sinalizam que o humor contagioso é um dos recursos dos líderes carismáticos para influenciar seus seguidores (Bono \& Ilies, 2006). Verifica-se que pessoas emocionalmente inteligentes são mais bem avaliadas por seus pares e/ou supervisores em termos de sensibilidade interpessoal, sociabilidade, interação positiva, contribuição para um ambiente de trabalho positivo e tolerância ao estresse (Groves, 2006; Lopes, Grewa, Kadis, Gall, \& Salovey, 2006).

A crescente ascensão da mulher a funções gerenciais e as crenças de que elas apresentam padrões de IE diferentes aos dos homens (Baron-Cohen, 2004) tornam justificáveis os estudos sobre as diferenças entre os dois sexos. O processo de socialização institui papéis sociais que influenciam na constituição do autoconceito de gênero com repercussões no papel profissional. Por exemplo, ao homem é exigido maior controle emocional, ao passo que à mulher incentiva-se o uso da sensibilidade. Nesse contexto se insere o objetivo principal deste estudo, que foi estabelecer uma comparação entre IE e CE de gestores de ambos os sexos e pertencentes a países distintos, visando compreender melhor o papel do sexo e do nível educacional no desempenho de funções gerenciais.

\section{INTELIGÊNCIA EMOCIONAL}

A inteligência emocional (IE) pode ser definida como um conjunto de habilidades que permitem identificar e compreender as emoções, saber gerenciá-las em si e nos outros e utilizá-las para melhorar a performance cognitiva (Mayer et al., 1999). Alguns autores as consideram como traços disposicionais (Mickolajczak \& Luminet, 2008; Petrides \& Furnham, 2000 b), enquanto outros a tratam como uma competência (a ser desenvolvida) e o resultado de crenças sobre o gerenciamento efetivo da emoção (autoconsciência, empatia, humor positivo e tomada de decisão)
(Ashkanasy \& Daus, 2005; Walter, Cole, \& Humphrey, 2011).

Não há consenso sobre o número de dimensões da IE. Alguns autores trabalham com uma única dimensão (Schutte et al., 1998), outros com três (Salovey, Mayer, Goldman, Turvey, \& Palfai, 1995) ou quatro dimensões (Mayer et al., 1999; Petrides \& Furnham, 2000a). O presente estudo tem em conta a medida unidimensional de Schutte et al., que apesar de unidimensional, baseou-se conceitualmente no modelo de três dimensões de Mayer e Salovey (1990): expressão, regulação e utilização das emoções.

A dimensão expressão faz referência à capacidade de expressar o que se sente, o que, de certo modo, facilita o contágio emocional do outro. A regulação emocional é definida como a capacidade de regular as emoções de acordo com o evento vivido. Dados empíricos sugerem que indivíduos de maiores pontuações em IE utilizam mais estratégias de ação profunda (mudança cognitiva), consideradas mais efetivas para o bem-estar, do que de ação superficial, isto é, supressão ou mudança da expressão, sem mudança de sentimento (Liu, Prati, Perrewé, \& Ferris, 2008). Finalmente, a dimensão utilização das emoções é definida como a efetividade no uso da informação emocional e inclui: planejamento flexível, pensamento criativo, atenção redirecionada e motivação (Schutte et al., 1998). Um estudo sobre o papel da emoção na tomada de decisões de negociadores concluiu que aqueles que empregaram estratégias de regulação de ação profunda tiveram melhor desempenho quando comparados aos que utilizaram estratégias de regulação de ação superficial (Fenton-O'Creevy, Soane, Nicholson, \& Willman, 2011).

Estudos sobre as relações entre sexo e IE não têm sido conclusivos. Porém, comumente, entende-se que este último é um atributo menos observado em contextos de trabalho masculino (Lindebaum \& Cassell, 2012), estando mais presente em mulheres (Day \& Carroll, 2004; Petrides, Furnham, \& Martin, 2004; Schutte et al., 1998), por serem mais preparadas socialmente para lidar com as emoções. Nessa direção, a pesquisa de Denham, Bassett e Wyatt (2010) concluiu que a socialização emocional de crianças em idade pré-escolar está relacionada com o sexo. As mães muitas vezes assumem o papel de portadoras da "função emocional" na família e as filhas parecem ser 
mais suscetíveis a assumir o mesmo padrão emocional. Embora alguns estudos sinalizem que as mulheres estão mais atentas às emoções que os homens (Kafetosis, 2004), outros apontam que os homens possuem mais capacidade de regulá-las (Lopes-Zafra, GarciaRetamero, \& Berrios Martos, 2012; Queirós, Fernandez-Berrocal, Extremera, Cancela Carral, \& Queirós, 2005).

\section{CONTÁGIO EMOCIONAL}

O contágio emocional (CE) pode ser definido como um processo de imitação inconsciente de expressões emocionais do outro, por meio de célulasespelho, produzindo uma experiência emocional simultânea e congruente com a original (Hatfield, Cacioppo, \& Rapson, 1993). A importância do CE reside em contribuir para a convergência de emoções entre dois ou mais atores sociais, fundamentais na função gerencial por possibilitar interações mais efetivas (Barsade, 2002; Doherty, Orimoto, Singelis, Hatfield, \& Hebb, 2005; George, 1990; Kelly \& Barsade, 2002; Rivera, 1992; Totterdell, Kellett, Teuchmann, \& Briner, 1998).

Em pesquisa experimental sobre o impacto do humor de líderes em membros do grupo, Sy, Côté e Saavedra (2005) concluíram que trabalhadores cujos líderes exibiram humores mais positivos experimentaram mais humor positivo do que os que interagiram com líderes com humor mais negativo. Este mecanismo de contágio também ficou evidenciado no tom afetivo grupal: grupos com líderes que apresentaram humor positivo experimentaram um tom afetivo também positivo. Contudo, há que dizer que as emoções não têm o mesmo poder de contágio. A propósito, estudo realizado por Gonzales, Castillo e Swinkelis (2002) sobre a percepção de emoções contagiosas apontou como mais prevalentes a felicidade, o entusiasmo ou estimulação, a tristeza, a raiva e o medo. Em estudo posterior, Formiga (2006) concluiu que as mulheres são mais expressivas para transmitir raiva, alegria e tristeza.

As pessoas mais propensas ao contágio emocional são aquelas que (a) prestam muita atenção e são capazes de decifrar as expressões emocionais dos outros, (b) se percebem interdependentes e vinculadas às demais pessoas, (c) tendem a imitar expressões faciais, vocais e posturais alheias e (d) são vulneráveis a comentários alheios sobre elas (Hatfield et al., 1993). Essas características parecem estar associadas a um perfil mais feminino, como identificado por BaronCohen (2004), que encontrou que mulheres têm maior tendência ao contágio emocional do que homens por serem mais empáticas e preocupadas com as relações interpessoais.

\section{RELACIONANDO INTELIGÊNCIA EMOCIONAL E CONTÁGIO}

A relação entre IE e contágio emocional tem sido pouco pesquisada. A IE pode ajudar a construir relacionamentos interpessoais mais harmoniosos, proporcionando bem-estar. O CE tem se revelado um importante mecanismo de influência de líderes em relação a seus seguidores, sendo preponderante para o seu desempenho. Os líderes diferem no quanto estão conscientes das experiências emocionais e no grau em que podem expressar de maneira verbal e não verbal suas emoções, contagiando seus seguidores (George, 2000); acentua-se que sinais não verbais parecem ter maior poder de contágio que os verbais (Barsade, 2002; Hatfield et al., 1993; Walter \& Bruch, 2008).

Em seu estudo sobre a paixão no comportamento do empreendedor, Cardon (2008) sugeriu que os empresários emocionalmente inteligentes são capazes de gerenciar as emoções próprias e as dos outros, assim como escolher as emoções positivas ou negativas que desejariam exibir a seus empregados. Pesquisas sinalizam haver diferenças não só individuais, mas culturais (Côté \& Hideg, 2011), de valores (Chen, French, \& Schneider, 2006) e de normas sociais (Pereira, 2009), que explicam a escolha de comportamentos e o julgamento sobre quais deles seriam os mais apropriados à situação. Na mesma linha de argumentação, Sutton e Rafaeli (1988) asseveram que as normas sociais interferem na regulação e expressão das emoções nos diversos ambientes sociais. Por exemplo, a supressão da emoção, ou seja, a inibição da expressão emocional, apesar de ser relacionada a consequências negativas (John \& Gross, 2003), também tem um papel de preservar sistemas culturais relacionados com a hierarquia (Matsumoto et al., 2008).

Em resumo, parece relevante conhecer como se relacionam a inteligência e o contágio emocionais, so- 
bretudo no contexto organizacional. Além disso, parece que esses construtos podem ser afetados pelo sexo das pessoas, e também pelo nível educacional e o exercício da função gerencial. Esses aspectos motivaram o presente estudo, que propõe comparar gerentes do Brasil e Angola nos construtos IE e CE, avaliando o papel das variáveis demográficas anteriormente mencionadas.

\section{MÉTODO}

\section{Participantes}

Participaram 431 trabalhadores, sendo 300 do Brasil (35\% gestores) e 131 de Angola (45\% gestores). A média geral de idade dos brasileiros $(m=29,2, d p=$ $9,97)$ foi menor que a dos angolanos $(m=32,7, d p=$ $7,18)$, sendo os gestores mais velhos tanto no Brasil $\left[\left(m_{\text {gestores }}=34,7, d p=10,09\right)\right.$ e $\left(m_{\text {não-gestores }}=26,2, d p=\right.$ $8,57)]$ quanto em Angola $\left[\left(m_{\text {gestores }}=35,1, d p=7,58\right) \mathrm{e}\right.$ $\left.\left(m_{\text {não-gestores }}=30,8, d p=6,25\right)\right]$. Em relação ao sexo, houve maior porcentagem de mulheres no Brasil $(65,7 \%)$ e em Angola (53,4\%). Quanto ao nível educacional, observou-se maior porcentagem de homens com ensino superior nos dois países (Brasil $=45 \%$ e Angola $=51 \%$ ) muito embora o quantitativo total com ensino superior fosse maior na amostra brasileira $(81,6 \%)$ do que na angolana $(48,1 \%)$. A amostra foi não probabilística.

\section{Instrumentos}

Além de quatro perguntas demográficas (sexo, idade, nível educacional e exercício da função gerencial), incluídas ao final de um questionário eletrônico, os participantes responderam a dois instrumentos:

Escala de Inteligência Emocional (EIS). Criada por Schutte et al. (1998), reúne 33 itens (e.g., Espero me sair bem na maioria das coisas que tento; Quando estou de bom humor, resolver problemas é fácil para mim) que definem uma estrutura unifatorial $(\alpha=$ $0,90)$. Tais itens são respondidos em escala tipo Likert, de cinco pontos, variando de 1 (Discordo fortemente) a 5 (Concordo fortemente). Estudo anterior sobre a estrutura fatorial desta medida sugeriu a exclusão de três itens, propondo uma versão com 30 itens, que foi adotada nesta pesquisa (Gouveia, Pessoa, Araújo, Soares, \& Guerra, 2012).
Escala de Contágio Emocional. Desenvolvida por Doherty (1997), a medida consta de 15 itens organizados em dois fatores: emoções positivas (e.g., amor, felicidade) $(\alpha=0,82)$ e emoções negativas (e.g., raiva, medo e tristeza) $(\alpha=0,80)$. Inclui ainda três itens para medir o nível de atenção dado às emoções de outras pessoas. O conjunto de itens é respondido em escala tipo Likert, de quatro pontos, variando de 1 (Sempre) a 4 (Nunca). Sua adaptação ao contexto brasileiro apontou uma estrutura de cinco fatores de primeira ordem (emoções básicas) e dois de segunda ordem, definidos como emoções positivas $[\alpha=0,64$; amor $(\alpha=0,56)$ e felicidade $(\alpha=0,52)]$ e emoções negativas $[\alpha=0,73$; medo $(\alpha=0,52)$, raiva $(\alpha=0,61)$ e tristeza $(\alpha=0,60)$ ] (Gouveia, Guerra, Santos, Rivera, \& Singelis, 2007). A versão, utilizada no presente estudo, excluiu os itens de atenção às emoções dos outros, visto que o foco de interesse era no tipo de emoção contagiada, positiva ou negativa.

Duas dimensões compõem a estrutura fatorial da escala de contágio na amostra brasileira: contágio para emoções positivas (Fator 1), com seis itens $(\alpha=0,66)$; e contágio para emoções negativas (Fator II), com oito itens $(\alpha=0,66)$. O conjunto de 14 itens apresentou valor correspondente de 0,75 (um item ambíguo foi excluído: Choro em filmes tristes). Em Angola, apesar de $K M O(0,68)$ e Teste de Esfericidade de Bartlett $\left(\chi^{2}=287,94, p<0,001\right)$ indicarem fatorabilidade da medida, a saturação dos itens não correspondeu ao pressuposto teórico, apresentando carga cruzada em cinco itens. Optou-se, então, por uma solução unifatorial $(\alpha=0,70)$ de 14 itens (Gondim, Gouveia, Simões, Luna, Morais, \& Saveia, 2014).

\section{Procedimento}

No Brasil os participantes receberam o convite para responder à pesquisa por $e$-mail, que continha um link para acessar a versão eletrônica do questionário. A pesquisa era iniciada mediante concordância com o Termo de Consentimento Livre e Esclarecido. Em virtude do acesso limitado a recursos informáticos, a coleta em Angola foi feita por pesquisadores locais de forma individual e coletiva; neste último caso, em salas com computadores. A todos foi informado que se tratava de uma pesquisa sobre emoções no exercício da função de gestão, não havendo respostas certas ou erradas. Assegurou-se a confidencialidade de suas 
respostas, e que estas seriam tratadas coletivamente. A coleta de dados realizou-se em 2010 no Brasil e 2011 em Angola. O tempo para o preenchimento do questionário foi de, aproximadamente, 30 minutos, não diferindo entre os países.

\section{Análise de Dados}

Os dados foram analisados com o SPSS (versão 18). Utilizaram-se estatísticas descritivas para caracterização dos participantes. Foram computadas análises dos fatores principais (rotação oblíqua) para as medidas de inteligência e contágio emocionais. As estruturas fatoriais resultantes consideraram as cargas fatoriais encontradas, a saturação nos fatores e, sobretudo, o sentido conceitual dos itens. Com o propósito de comparar os países, calcularam-se testes $t$ para amostras independentes e análises de variância para avaliar efeitos principais e de interação de variáveis demográficas (país, nível educacional, função e sexo) em relação à inteligência e ao contágio emocional. Ao final, correlações (Pearson) entre os construtos foram calculadas.

\section{RESULTADOS}

São apresentados a seguir os resultados de inteligência e contágio emocionais nas duas amostras, levando em conta as variáveis: função gerencial, sexo e nível educacional. Primeiramente, as soluções fatoriais dos construtos são descritas para cada amostra, passando em seguida a comparar suas pontuações em função do país e das demais variáveis. Por último, avaliam-se os efeitos, principal e de interação, dessas variáveis, findando com as correlações entre os construtos de interesse.

\section{A Inteligência Emocional de Brasileiros e Angolanos}

Foram realizadas análises de fatores principais para as amostras do Brasil e Angola. Apesar de Petrides e Furnham (2000a) terem proposto a adaptação do construto de Schutte et al. (1998) a um modelo de quatro fatores (habilidades sociais, avaliação das emoções, otimismo/regulação do humor e utilização das emoções) e Gouveia et al. (2012) terem confirmado este modelo, os itens não saturaram como esperado. Dessa maneira o modelo multifatorial não se apresentou como a melhor solução encontrada, optando-se pela estrutura unidimensional como previsto no modelo original de Schutte et al. (1998).

No Brasil a solução unifatorial $(\alpha=0,82)$ explicou $18,8 \%$ da variância total. Dos 30 itens da medida, 21 saturaram com cargas fatoriais entre 0,40 e 0,53 . Em Angola também se encontrou uma solução unifatorial $(\alpha=0,85)$, que explicou $20,4 \%$ da variância, reunindo 20 itens cujas saturações variaram de 0,41 a 0,65. Objetivando a comparação dos países nesta medida, foram retirados dois itens no Brasil [Itens 19 (Sei por que minhas emoções mudam) e 25 (Estou consciente das mensagens verbais que as outras pessoas enviam)] e um em Angola [Item 10 (Espero que coisas boas aconteçam)], mantendo a confiabilidade das escalas dos dois países praticamente inalterada: Brasil $(\alpha=$ $0,81)$ e Angola $(\alpha=0,84)$. Assim, a escala ficou composta por 19 itens.

Compararam-se as pontuações totais de IE de cada país tendo sido constatada diferença $[t(429)=3,57, p$ $<0,001]$, apresentando as seguintes pontuações: Brasil $(m=3,9, d p=0,38)$ e Angola $(m=4,1, d p=0,39)$. No Brasil, observaram-se efeitos principais do sexo $\left[F_{(1}\right.$, 299) $=3,88, p<0,05]$, em que as mulheres $(m=3,93, d p$ $=0,41)$ apresentaram maior IE que os homens $(m=$ $3,87, d p=0,32)$, e da função exercida $\left[F_{(1,299)}=8,82\right.$, $p<0,05]$, em que os gestores $(m=4,0, d p=0,42)$ apresentaram maior inteligência que os não gestores ( $m=3,85, d p=0,35)$. Não foram observados quaisquer efeitos de interação entre as variáveis demográficas. Em Angola, constatou-se efeito de interação marginal entre as variáveis sexo e nível educacional $\left[F_{(1}\right.$, $\left.{ }_{123)}=3,17, p=0,07\right]$. Especificamente, evidenciou-se que mulheres de nível educacional superior ( $m=4,2$, $d p=0,35)$ apresentam médias maiores de IE do que homens com ensino médio ( $m=4,07 d p=0,26)$, seguido de homens com ensino superior $(m=4,0 d p=$ $0,52)$ e mulheres com ensino médio ( $m=3,9, d p$ $=0,27$ ). Os resultados sugerem que o sexo e o nível educacional podem ter um efeito direto positivo, sobre os níveis de IE entre mulheres de Angola.

\section{Contágio Emocional entre Brasileiros e Angolanos}

Um estudo sobre as relações entre o contágio emocional e o sentimento de constrangimento em amostras de trabalhadores brasileiros e angolanos (Gondim et al., 2014) concluiu não haver diferença quanto a 
contágio emocional nos dois países. Observou-se efeito principal do sexo, evidenciando que as mulheres apresentam maior contágio emocional nos dois países.

Em relação ao contágio de emoções negativas, observou-se apenas efeito principal do sexo reafirmando-se que mulheres são mais susceptíveis ao contágio que homens. Apenas as emoções positivas apresentaram efeito de interação entre nível educacional, função gerencial e sexo, em que o nível educacional modificou a relação entre as outras duas variáveis.

\section{Correlacionando Inteligência Emocional e Contágio}

No Brasil, a correlação entre as pontuações totais de IE e CE foi positiva, porém fraca $(r=0,29, p<$ $0,001)$. Em Angola o resultado seguiu a mesma direção, embora apresentando relação mais forte $(r=0,37$, $p<0,001)$. Quanto à correlação da pontuação total de IE com as duas dimensões de CE no Brasil, observouse que esta se correlacionou direta e moderadamente com as emoções positivas ( $r=0,38, p<0,001$ ), sendo mais fraca sua correlação com as emoções negativas $(r=0,16, p<0,001)$.

\section{DISCUSSÃO}

O objetivo deste artigo foi comparar trabalhadores brasileiros e angolanos em relação à IE e ao contágio emocional, levando em conta as seguintes variáveis demográficas: função gerencial, sexo e nível educacional.

Inicialmente, é preciso dizer que, em ambos os países, a IE pode ser adequadamente representada por uma solução unifatorial, coerente com a proposição de Schutte et al. (1998). Porém, o CE mostrou diferenças quanto à estrutura: bidimensional no Brasil e unifatorial em Angola. Não se encontrou uma explicação teórica para esses achados, questionáveis devido à reduzida amostra em Angola. Estudos futuros nesse contexto poderão esclarecer tais resultados.

De modo geral, os achados permitem afirmar que os dois países apresentam comportamentos semelhantes quanto ao contágio emocional, mas diferenciam-se levemente nos níveis de inteligência emocional. Aspectos culturais talvez estejam na base dessa similaridade no $\mathrm{CE}$ e na pouquíssima diferença em IE, uma vez que os dois países apresentam traços tipicamente coletivistas como o sentimento de obrigação pelo outro e a priorização de metas grupais (Gouveia \& Clemente, 2000; Hofstede, 1984). Destaca-se que as médias de Brasil e Angola em IE foram altas; a pequena diferença entre elas pode estar relacionada à aludida orientação coletivista apontada por Hofstede, que faz com que se compartilhem valores sociais parecidos, tornando as culturas semelhantes em alguns aspectos.

Com relação à função gerencial, ficou evidente que no Brasil os gestores têm maior inteligência emocional que os não gestores. Isso pode ser explicado pela expectativa do papel profissional, em que é esperado do gestor estar mais preparado para lidar com as suas emoções e as de sua equipe, visto que o seu desempenho depende em grande parte das interações sociais que constrói (Fenton-O’Creevy et al., 2011). Essa prontidão também inclui o uso adequado do tipo de emoção a ser expressa ou exibida para cativar a equipe (Cardon, 2008; Côtè \& Hideg, 2011).

Ao supor que as organizações estabelecem regras de exibição de emoções (Liu et al., 2008) e aderem a normas culturais (Chen et al., 2006) descritivas e prescritivas (Pereira, 2009), é compreensível a tendência de o gestor no Brasil se adequar ao papel que lhe é imposto. Por exemplo, a mulher gestora inclui em sua postura alguns atributos masculinos e o homem, por sua vez, adota o caminho contrário, ampliando seu repertório emocional, o que contribui para que sua equipe o avalie como um líder eficaz (Groves, 2006). Ademais, em culturas coletivistas, deveres e obrigações são formalizados no exercício de papéis (Gouveia \& Clemente, 2000), incentivando homens e mulheres a realizarem adaptações necessárias para sua consecução.

Em Angola, foi observado efeito de interação entre o sexo e o nível educacional na inteligência emocional, onde mulheres com ensino superior obtiveram maiores médias em relação aos homens com nível educacional médio. Duas explicações podem ser dadas para esses achados. A primeira é que o ensino superior aumenta a habilidade de lidar com as emoções (Day \& Carroll, 2004), isto é, aumenta a capacidade de identificar similaridades e diferenças entre as emoções e seus impactos no próprio desempenho e no dos demais (Boyatzis et al., 1999; Mayer et al., 1999). A segunda explicação diz respeito ao próprio processo de socialização da mulher, que é mais preparada para 
lidar com a emoção (Baron-Cohen, 2004), assumindo a "função emocional" no contexto familiar (Bretherton, Lambert, \& Golby, 2005; Denham et al., 2010).

Os resultados sobre o contágio emocional evidenciam que nos dois países as mulheres se contagiam mais que os homens, o que é coerente com a teorização e as evidências descritas por Baron-Cohen (2004). Em direção congruente, Denham et al. (2010) observaram que, mais do que os filhos, as filhas parecem suscetíveis à socialização parental de competências emocionais.

No Brasil, o nível educacional parece modificar a interação entre sexo e função no tocante ao contágio a emoções positivas. Nesse caso, o ensino superior parece repercutir de modo distinto em homens e mulheres: o aumento do nível educacional do homem gestor faz aumentar a susceptibilidade ao contágio, ocorrendo o inverso para a mulher na mesma função. Com maior habilidade no manejo de emoções, a mulher talvez reconheça sua maior susceptibilidade ao contágio de emoções positivas e, ao exercer uma função gerencial, tende a adotar comportamentos emocionais considerados mais masculinos para se afirmar profissionalmente.

Por outro lado, a socialização masculina privilegia mais a racionalidade e a neutralidade do que o envolvimento afetivo, levando o homem a investir mais em interações sociais. Ao prestar mais atenção aos aspectos emocionais, ele pode obter maior sincronismo emocional com a equipe de trabalho. A demanda atual pela qualidade da interação entre líderes e sua equipe (Lopes et al., 2006; O’Boyle, Humphrey, Pollack, Hawver, \& Story, 2011) pode contribuir para que o líder seja mais sensível às emoções alheias, aumentando seu poder de influência sobre os seguidores. De fato, há estudos que sinalizam a importância do contágio na emergência de comportamentos cooperativos de seguidores (Barsade, 2002; Sy et al., 2005).

Embora não se tenha uma explicação precisa para o fato de, na amostra brasileira, as mulheres terem apresentado maior contágio a emoções negativas superior aos dos homens, é provável que isso se deva, em parte, à socialização de gênero feminino. Mulheres são mais sensíveis ao sofrimento alheio, o que repercutiu nas médias mais altas das emoções tristeza, medo e raiva (Gondim et al., 2014). Resultados que corroboraram os estudos de Formiga (2006), onde mulheres tiveram médias mais elevadas em emoções de raiva e tristeza, e de Gonzales et al. (2002) que identificaram a tristeza, a raiva e o medo como as emoções com maior poder de contágio.

$\mathrm{O}$ esforço de buscar associações entre IE e CE levando em conta o sexo, o nível educacional e o exercício da função de gestão, decorreu da suposição de que a inteligência emocional auxilia no desempenho dos gestores. Pressupõe-se, assim, que o contágio torna o gestor mais sensível às emoções de sua equipe de trabalho. Os resultados dos dois países permitem concluir que a mulher parece ser mais suscetível ao contágio, sendo também mais inteligente emocionalmente, o que aumenta sua probabilidade de ser sensível às demandas da equipe de trabalho. Neste âmbito, o aumento do nível educacional da mulher parece ter papel importante na inteligência emocional, como ficou evidenciado em Angola.

É importante assinalar que uma das limitações potenciais deste estudo foi o número reduzido de participantes em Angola, o que pode ter influenciado nas estruturas fatoriais encontradas, como mencionado anteriormente. O limitado acesso aos recursos de informática em Angola também dificultou a ampliação da amostra. Por fim, instrumentos de autorrelato trazem limites para a generalização do estudo, em virtude da tendência de os participantes responderem às questões levando em conta o que é socialmente aceito (desejabilidade social), esquivando-se de dizer o que realmente pensam (Mikolajczak, Petrides, Coumans, \& Luminet, 2009). Desta forma, outras estratégias de investigação complementares podem ser adotadas, como o uso de recursos iconográficos, neuroimagem e associações implícitas. Observações de campo também poderão ser úteis para compreender melhor os construtos de interesse, que são susceptíveis à influência cultural e à socialização de gênero.

\section{REFERÊNCIAS}

Ashkanasy, N. M., \& Daus, C. S. (2005). Rumors of the death of emotional intelligence in organizational behavior are vastly exaggerated. Journal of Organizational Behavior, 26, 441-452.

Barbalet, J. (2011). Emotions beyond regulation: Backgrounded emotions in science and trust. Emotion Review, 3, 36-43.

Baron-Cohen, S. (2004). Diferença essencial: A verdade sobre o cérebro de homens e mulheres (N. Capelo, Trans.). Rio de Janeiro: Objetiva. 
Barsade, S. G. (2002). The ripple effect: Emotional contagion and its influence on group behavior. Administrative Science Quarterly, 47, 644-675.

Bono, J. E., \& Ilies, R. (2006). Charisma, positive emotions, and mood contagion. The Leadership Quarterly, $17,317-334$

Boyatzis, R., Goleman, D., \& Rhee, K. S. (1999). Clustering competence in emotion intelligence: Insights from the Emotional Competence Inventory (ECI). In R. BarOn, \& J. D. A. Parker (Eds.), Handbook of emotional intelligence (pp. 2-36). San Francisco, CA: Jossey-Bass.

Bretherton, I., Lambert, J. D., \& Golby, B. (2005). Involved fathers of preschool children as seen by themselves and their wives: Accounts of attachment, socialization, and companionship. Attachment \& Human Development, 7 , 229-251.

Campos, J. J., Walle, E. A., Dahl, A., \& Main, A. (2011). Reconceptualizing emotion regulation. Emotion Review, 3, 26-35.

Cardon, M. S. (2008). Is passion contagious? The transference of entrepreneurial passion to employees. Human Resource Management Review, 18, 77-86.

Chen, X., French, D. C., \& Schneider, B. H. (2006). Culture and peer relationships. In X. Chen, D. R. French, \& B. H. Schneider (Eds.), Peer relationship in cultural context (pp. 3-22). New York: Cambridge.

Côtè, S., \& Hideg, I. (2011). The ability to influence others via emotion displays: A new dimension of emotional intelligence. Organizational Psychology Review, 1, 53-71.

Day, A. L., \& Carroll, S. A. (2004).Using an ability-based measure of emotional intelligence to predict individual performance, group performance, and group citizenship behavior. Personality and Individual Differences, 36, 1.443-1.458.

Denham, S. A., Basset, H. H., \& Wyatt, T. M. (2010). Gender differences in the socialization of preschoolers' emotional competence. In A. Kennedy Root \& S.A. Denham (Eds.), The role of gender in the socialization of emotion: Key concepts and critical issues. New directions for child and adolescent development (pp. 29-49). San Francisco, CA: Jossey-Bass.

Doherty, R. W. (1997). The Emotional Contagion Scale: A measure of individual differences. Journal of Nonverbal Behavior, 21, 131-154.

Doherty, R. W, Orimoto, L., Singelis, T .M., Hatfield, E., \& Hebb, J. (2005). Emotional contagion: Gender and occupational differences. Psychology of Women Quarterly, $19,355-371$.

Fenton-O'Creevy, M., Soane, E., Nicholson, N., \& Willman, P. (2011). Thinking, feeling and deciding: The influence of emotions on the decision making and performance of traders. Journal of Organizational Behavior, 32, 1.044-1.061.

Formiga, N. S. (2006). Diferença de gênero nos antecedentes das emoções de raiva, alegria e tristeza. Revista Cien- tífica Eletrônica de Psicologia, IV. Recuperado em 26 de fevereiro de 2012, de http://www.revista.inf.br/ psico logia06/ pages/artigos/psic-edic06-anoiii-art03.pdf.

George, J. M. (1990). Personality, affect, and behavior in groups. Journal of Applied Psychology, 75, 107-116.

George, J. M. (2000). Emotions and leadership: The role of emotional intelligence. Human Relations, 53, 10261055.

Gondim, S. M. G., Gouveia, V. V., Simões, A. C. A., Luna, A. F., Morais, F. A., \& Saveia, J. M. (2014). Constrangimento, contágio emocional e gestão: Um estudo transcultural. Revista de Psicologia da UFC, 5, 7-20.

Gonzales, J., Castillo, M., \& Swinkeles, A. (2002). Perceptions of contagious emotions and contagious people. PSI CHI Journal of Undergraduate Research, 7, 185-191.

Gouveia, V., \& Clemente, M. (2000). O individualismocoletivismo no Brasil e na Espanha: Correlatos sociodemográficos. Estudos de Psicologia, 5, 317-346.

Gouveia, V. V., Guerra, V. M., Santos, W. S., Rivera, G. A., \& Singelis, T. M. (2007). Escala de Contágio Emocional: Adaptação ao contexto brasileiro. Psico-PUCRS, $79,45-54$

Gouveia, V. V., Pessoa, V. S., Araújo, R. C. R., Soares, A. K. S., \& Guerra, V. M. (2012). Emotional Intelligence Scale: Adaptation and evidences of validity and reliability. Manuscrito submetido à publicação.

Gross, J. J., \& Barret, L. F. (2011). Emotion Generation and Emotion Regulation: One or Two Depends on your Point of View. Emotion Review, 3, 8-16.

Groves, K. S. (2006). Leader emotional expressivity, visionary leadership, and organizational change. Leadership and Organizational Development Journal, 27, 566583.

Hatfield, E., Cacioppo, J. T., \& Rapson, R. L. (1993). Emotional contagion. Current Directions in Psychological Science, 2, 96-99.

Hochschild, A. R. (1983). The managed heart: Commercialization of human feeling. Berkeley, CA: University of California Press.

Hofstede, G. (1984). Culture's consequences: International differences in work-related values. Beverly Hills, CA: Sage.

John, O. P., \& Gross, J. J. (2003). Individual Differences in Two Emotion Regulation Processes: Implications for Affect, Relationships, and Well-Being. Journal of Personality and Social Psychology, 85, 348-362.

Kafetosis, K. (2004). Attachment and emotional intelligence abilities across the life course. Personality and Individual Differences, 37, 129-145.

Kelly, J. R., \& Barsade, S. G. (2002). Mood and emotions in small groups and work teams. Organizational Behavior and Human Decision Processes, 86, 99-130.

Lindebaun, D., \& Cassell, C. (2012). A Contradiction in Terms? Making Sense of Emotional Intelligence in a 
Construction Management Enviroment. British Journal of Management, 23, 65-79.

Liu, Y., Prati, M. L., Perrewé, P. L., \& Ferris, G. R. (2008).The relationship between emotional resources and emotional labor: An exploratory study. Journal of Applied Social Psychology, 38, 2.410-2.439.

Lopes, P. N., Grewa, D., Kadis, J., Gall, M., \& Salovey, P. (2006). Evidence that emotional intelligence is related to job performance and affect and attitudes at work. Psicothema, 18, 132-138.

Lopes-Zafra, E., Garcia-Retamero, R., \& Berrios Martos, M. P. (2012). The relationship between transformational leadership and emotional intelligence from a gendered approach. The Psychological Record, 62, 97-114.

Matsumoto, D., Yoo, S. H., Nakagawa, S., Alexandre, J., Altarriba, J., Anguas-Wong, A. M., Zengeya, A. (2008). Culture, emotion regulation, and adjustment. Journal of Personality and Social Psychology, 94, 925-937.

Mayer, J., Caruso, D. R, \& Salovey, P. (1999). Emotional intelligence meets traditional standards for an intelligence. Intelligence, 27, 267-298.

Mickolajczak, M., \& Luminet, O. (2008). Trait emotional intelligence and the cognitive appraisal of stressful events: An exploratory study. Personality and Individual Differences, 44, 1445-1453.

Mickolajczak, M., Petrides, K. V., Coumans, N., \& Luminet O. (2009). The moderating effect of trait emotional intelligence on mood deterioration following laboratoryinduced stress. International Journal of Clinical and Health Psychology, 9, 455-477.

Nelis, D., Quoidbach, J., Hansenne, M., \& Mickolajczak, M. (2011). Measuring individual differences in emotion regulation: The Emotion Regulation Profile-Revised (ERP-R). Psychologica Belgica, 5, 49-91.

Niven, K., Totterdel, P., Stride, C. B., \& Holman, D. (2011). Emotion Regulation of Others and Self (EROS): The development validation of a new individual differrence measure. Current Psychology, 30, 53-73.

O’Boyle, E. H. Jr., Humphrey, R. H., Pollack, J. M, Hawver, T. H., \& Story, P. A. (2011). The relationship between emotional intelligence and job performance: A meta-analysis. Journal of Organizational Behavior, 32, 788-818.

Pereira, A. S. L. S. (2009). Normas sociais, crenças sobre a natureza da homossexualidade e preconceito contra os homossexuais (Tese de Doutorado). Instituto Universitário de Lisboa, Lisboa.

Petrides, K. V., \& Furnham, A. (2000a). On the dimension structure of emotional intelligence. Personality and Individual Differences, 29, 313-320.

Petrides, K. V., \& Furnham, A. (2000b). Gender differences in measured and self-estimated trait emotional intelligence. Sex Roles, 42, 449-461.
Petrides, K. V., Furnham, A., \& Martin, G. N. (2004). Estimates of emotional and psychometric intelligence: Evidence for gender-based stereotypes. Journal of Social Psychology, 144, 149-162.

Queirós, M. M., Fernández-Berrocal, P., Extremera, N., Cancela Carral, J. M., \& Queirós, P. S. (2005). Validação e fiabilidade da versão portuguesa modificada da Trait Meta-Mood Scale. Revista de Psicologia, Educação e Cultura, 9, 199-216.

Rivera, J. D. (1992). Emotional climate: Social structure and emotional dynamics. International Review of Studies of Emotion, 2, 197-218.

Salovey, P., \& Mayer, J. D. (1990). Emotional intelligence. Imagination, Cognition, and Personality, 9, 185-211. Obtido de http://www.unh.edu/emotional_intelligence/ EI\%20Assets/Reprints...EI\%20Proper/EI1990\%20Emo tional\%20Intelligence.pdf

Salovey, P., Mayer, J. D., Goldman, S. L., Turvey, C., \& Palfai, T. P. (1995). Emotional attention, clarity, and repair: Exploring emotional intelligence using the Trait Meta Mood Scale. In J. W. Pennebaker (Ed.), Emotion, disclosure, and health (pp. 125-151). Washington, DC: American Psychology Association.

Schutte, N. S., Malouff, J. M., Hall, L. E., Haggerty, D. J., Cooper, J. T., Golden, C. J., \& Dornheim, L. (1998). Development and validation of a measure of emotional intelligence. Personality and Individual Differences, 25, 167-177.

Sutton, R. I., \& Rafaeli, A. (1988).Untangling the relationship between displayed emotions and organizational sales: The case of convenience stories. The Academy of Management Journal, 32, 481-487.

Sy, T., Côté, S., \& Saavedra, R. (2005). The contagious leader: Impact the leader's mood on the mood of group members, group affective tone, and group processes. Journal of Applied Psychology, 90, 295-305.

Tamir, M. (2011). The maturing field of emotion regulation. Emotion Review, 3, 3-7.

Totterdell, P., Kellett, S., Teuchmann, K., \& Briner, R. B. (1998). Evidence of mood linkage in work groups. Journal of Personality and Social Psychology, 74, 1.5041.515 .

Walter, F., \& Bruch, H. (2008). The positive group affect spiral: A dynamic model of the emergence of positive affective similarity in work groups. Journal of Organizational Behavior, 29, 239-261.

Walter, F., Cole, M. S., \& Humphrey, R. H. (2011). Emotional intelligence: Sine qua non of leadership or folderol. Academy Management Perspectives, 25, 46-59.

Recebido em: 12/02/2013 Revisões requeridas em: 01/05/2013 Aceito em: 13/05/2013 